\title{
Confinement of a Nonionic Surfactant Membrane Within a Montmorillonite as a New Way to Prepare Organoclay Materials
}

\author{
Régis Guégan ${ }^{a *}$, Marcelo Giovanela ${ }^{b}$ \\ ISTO, UMR 7327, CNRS, Université d'Orléans, 1 A Rue de la Férollerie, 45071 Orléans Cedex 2, \\ France \\ ${ }^{b}$ Centro de Ciências Exatas e da Tecnologia, Universidade de Caxias do Sul, 95070-560 Caxias do Sul, \\ RS, Brazil
}

Received: May 10, 2016; Revised: August 01, 2016; Accepted: September 04, 2016

\begin{abstract}
The aim of this study was to prepare and characterize a hybrid layered material (organoclay) with a Na-montmorillonite and the triethylene glycol mono-n-decyl ether $\left(\mathrm{C}_{10} \mathrm{E}_{3}\right)$ nonionic surfactant which forms a lamellar phase at room temperature. The synthesized organoclay was characterized by complementary techniques (Fourier transform infrared spectroscopy and X-ray diffraction). Experiments in conjunction with electron density analysis showed that a bilayer or membrane of $\mathrm{C}_{10} \mathrm{E}_{3}$ was intercalated within the interlayer space of a naturally exchanged $\mathrm{Na}$-montmorillonite. The intercalation of a bilayer of $\mathrm{C}_{10} \mathrm{E}_{3}$ in a clay mineral offers new perspectives for the manufacturing of nanomaterials. While showing a hydrophobic surface and a large interlayer space value, the resulting organoclay preserves the compensating cations within the interlayer space allowing one to perform ion exchanges, making easier the intercalation of further organic molecules of important size with functional properties or for environmental purposes.
\end{abstract}

Keywords: hybrid layered materials, clay minerals, organoclays, nonionic surfactant

\section{Introduction}

The organization of molecules in anisotropic thin films for the development of new nanocomposites is still an audacious challenge. One process of synthesis is the use of layered porous materials such as Layered Double Hydroxides (LDHs) or smectite as host structures for the orientation of guest molecules ${ }^{1-3}$.

Smectite clay minerals such as Wyoming montmorillonite display, in presence of water, cation exchange capacities (CEC), swelling, hydration properties and a high surface area which lead to strong adsorption properties. These properties have been highlighted in several applications involving catalysis, nanocomposite materials, geochemical barriers in the case of waste landfills as well as potential sorbents for water remediation ${ }^{1-8}$. The structure of dioctahedral smectite is represented by two $\mathrm{Si}_{4} \mathrm{O}_{10}$ tetrahedral sheets of sandwiching an aluminum $\left(\mathrm{Al}_{4}(\mathrm{OH})_{12}\right)$ or a magnesium $\left(\mathrm{Mg}_{6}(\mathrm{OH})_{12}\right)$ octahedral sheet. Ionic substitution can occur in the octahedral sheet or less frequently in the tetrahedral sheet, which leads to a negatively charged clay layer. The charge equilibrium is counterbalanced by exchangeable cations such as $\mathrm{Na}^{+}, \mathrm{Ca}^{2+}, \mathrm{K}^{+}$or $\mathrm{Mg}^{2+}$ in the interlayer. However, in the presence of water, the interactions between silicate layers and cations are modified. The cations are surrounded by water and the interlayer space is expanded allowing the adsorption of several layers of water.

* e-mail: regis.guegan@univ-orleans.fr
The adsorption process of guest molecules on smectite clay minerals mainly supports an ion exchange with the interlayer cations or counterions ${ }^{1-3}$. Many studies have been devoted to the synthesis of cationic organoclays ${ }^{1-3}$. These materials are obtained after an ion exchange with quaternary ammonium cationic surfactant. The resulting hybrid layered material exhibits a change of its chemical nature that switches from hydrophilic to hydrophobic. These modifications allow the adsorption of neutral organic compounds and offer numerous applications ${ }^{1,7-9}$. The first is relied to environmental issue, where many authors suggested the use of organoclays as geochemical layers for the capture of hydrophobic pollutants which usually pass through the natural clay barrier implemented against pollution, or as sorbent materials for water remediation. Other applications are the use of organoclays as basis for the implementation of polymer layered nanocomposites, where nanocomposite properties such as biodegradability, impermeability, and heat resistance are highly improved ${ }^{7,10}$.

Several authors have recently raised the interest to synthesize organoclays by the use of other surfactants ${ }^{1,11-13}$. Nonionic surfactants containing $n-\mathrm{C}_{\mathrm{n}} \mathrm{H}_{2 \mathrm{n}+1}\left(\mathrm{OCH}_{2} \mathrm{CH}_{2}\right)_{\mathrm{m}} \mathrm{OH}$ (abbreviated as $\mathrm{C}_{\mathrm{n}} \mathrm{E}_{\mathrm{m}}$ ) can self-assemble to form bicontinuous structures made of amphiphilic bilayers ${ }^{14}$. In particular, the $\mathrm{C}_{10} \mathrm{E}_{3}$ (tri-ethylene glycol mono n-decyl ether) and water system forms internal structures organized by surfactant bilayers such as the lamellar $\left(\mathrm{L}_{\alpha}\right)$ characterized by a stack of surfactant molecules aggregated into a two dimensional structure $^{12-15}$. The intercalation of a $\mathrm{C}_{10} \mathrm{E}_{3}$ bilayer within the 
interlayer space of a Na-montmorillonite, resulting in the state of the lyotropic liquid crystal in a lamellar phase points out the possible link between bulk surfactants molecular structures and the way they pack in the internal structure of the clay mineral ${ }^{12-14}$. This last observation on nonionic organoclays differs from previous studies showing that nonionic surfactants adsorption is usually limited to lateral monolayers parallel to phyllosilicate surface ${ }^{11}$. The intercalation of a nonionic bilayer within the interlayer space of a Na-montmorillonite revives the interest to prepare organoclays with nonionic surfactants since they provide better both adsorption capacity and chemical stability than cationic analogous ${ }^{1}$.

Thus, the aim of this study is to characterize an organoclay synthesized with a $\mathrm{C}_{10} \mathrm{E}_{3}$ nonionic surfactant in a lamellar phase by complementary experimental techniques, including $\mathrm{X}$-ray diffraction in conjunction with electron density analysis, and Fourier transform infrared spectroscopy (FTIR).

\section{Materials and methods}

\subsection{Starting materials}

The Na-montmorillonite used in this study was supplied from the Clay Minerals Society. This clay mineral originates from the Newcastle formation (cretaceous), Crook County, Wyoming. The formula of the montmorillonite can be expressed as $\left(\mathrm{Ca}_{0.12} \mathrm{Na}_{0.32} \mathrm{~K}_{0.05}\right)\left[\mathrm{Al}_{3.01} \mathrm{Fe}(\mathrm{III})_{0.41} \mathrm{Mn}_{0.01} \mathrm{Mg}_{0.54}\right.$ $\left.\mathrm{Ti}_{0.02}\right]\left[\mathrm{Si}_{7.98} \mathrm{Al}_{0.02}\right] \mathrm{O}_{20}(\mathrm{OH})_{4}$, as calculated from its chemical composition. Sodium exchanges were performed by immersing the clay into a $1.0 \mathrm{~mol} \mathrm{~L}^{-1}$ solution of sodium chloride. The cation exchange was completed by washing and centrifuging five times with dilute saline solutions. Samples were finally washed with distilled-deionized water and transferred into dialysis tubes to chloride-free clays, being then dried in an oven at $60{ }^{\circ} \mathrm{C}$ for $48 \mathrm{~h}$.

The $\mathrm{C}_{10} \mathrm{E}_{3}$ surfactant has a purity better than $99.8 \%$ and was purchased from Nikko Chemicals, Inc., (Tokyo, Japan), being used without further purification (Figure 1). Distilled and deionized water was used to prepare the aqueous solutions of surfactant. The $\mathrm{C}_{10} \mathrm{E}_{3}$-water system self-assembles at room temperature in a lamellar phase of which bilayer thickness is assumed to be close to $27.6 \AA^{12-15}$.

\subsection{Synthesis of the organoclay composite}

A solution of $\mathrm{C}_{10} \mathrm{E}_{3}$ with distilled and deionized water was prepared at room temperature at $1.0 \times 10^{-2} \mathrm{~mol} \mathrm{~L}^{-1}$, above the critical micellar concentration (CMC) experimentally determined at $7.5 \times 10^{-4} \mathrm{~mol} \mathrm{~L}^{-1}$, displaying a lamellar phase as revealed by both optical and diffraction data ${ }^{15}$. The solution was homogenized at $500 \mathrm{rpm}$ using a magnetic stir bar at room temperature for several days in order to reach an equilibrium state, which was properly checked by phase contrast microscopy measurements as it was explained in

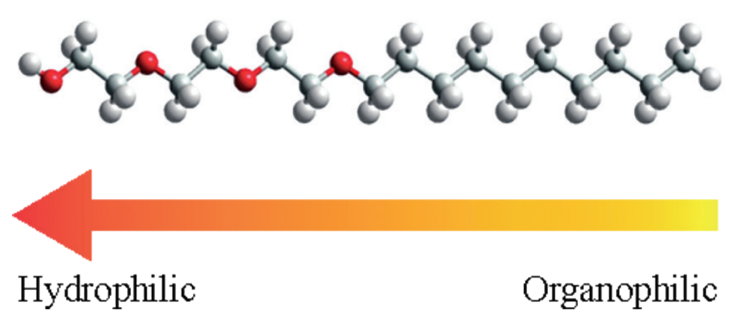

Figure 1: Representation of the $\mathrm{C}_{10} \mathrm{E}_{3}$ nonionic tensioactive molecule where the oxygen, carbon and hydrogen atoms are in red, gray and white colors, respectively.

more details elsewhere ${ }^{15}$. A clay suspension solution was then poured into the surfactant solution. The average $\mathrm{pH}$ value of the solution was about $6.5 \pm 0.2$ and remained constant during the synthesis. The obtained suspension was then stirred for $24 \mathrm{~h}$ at $250 \mathrm{rpm}$. The synthesized organoclay, separated by centrifugation, was twice rinsed with distilled water and dried at $80^{\circ} \mathrm{C}$ for $48 \mathrm{~h}$ before being crushed using an agate mortar.

\subsection{Characterization techniques}

Infrared spectra, in the region of $650-4000 \mathrm{~cm}^{-1}$, were measured by a Thermo Nicolet 6700 infrared spectrometer equipped with a Germanium attenuated total reflection (ATR) and deuterated triglycine sulfate (DTGS) detector. Each spectrum was the average of 128 scans collected at $2 \mathrm{~cm}^{-1}$ resolution. Organoclay and Na-montmorillonite samples were in powder form and were deposited on a germanium crystal and pressed by an aluminum anvil whereas a droplet of pure $\mathrm{C}_{10} \mathrm{E}_{3}$, in its lamellar phase (liquid form), was deposited on the ATR crystal.

XRD diffractograms of the Na-montmorillonite and $\mathrm{C}_{10} \mathrm{E}_{3}$ organoclay samples were measured using an ARL X'TRA Diffractometer (Thermo Electron Corporation, Ecublens, Switzerland) operating at $40 \mathrm{kV}$ and $40 \mathrm{~mA}$. The available scattering vector q range was between $7.11 \times 10^{-2} \AA^{-1}$ and 4.3 $\AA^{-1}$. This scattering vector q is calculated by Equation (1):

$$
q=\frac{4 \pi \sin \theta}{\lambda}
$$

where $2 \theta$ is the scattering angle and $\lambda=1.5405 \AA\left(\mathrm{CuK}_{\alpha}\right.$ radiation). Measurements were carried out at room temperature in the step-scanning mode with a step size of $3.55 \times 10^{-3} \AA^{-1}$.

\subsection{Electron density distribution}

The electron density distributions along the c-axis in the alternatively stacked layer materials were calculated by means of a series of $(00 l)$ peaks of high periodicity. On the basis of the electron density distribution, which is closely associated with the atom dispositions, the organoclays were characterized to exhibit a high regularity along the c-axis. 
The electron density, in projection along the normal of the layers, of the hybrid materials, was independently estimated with the calculations of Equation (2) and the Fourier transformations of the $(00 l)$ peaks, where $l, F(l), d, \varphi, x$, and $I$ denote the peak number $(00 l)$ of the diffraction order, the structural factor, layer distance, phase, the coordinate along the perpendicular direction of the layer, and the peak area, respectively.

$$
\rho(\chi)=\sum_{l=1}^{l_{\max }} F(l) \cos \left(\frac{2 \pi l x}{d}\right)
$$

where

$$
F(l)=\exp \{i \varphi(l)|\sqrt{I(l)}|\}
$$

\section{Results and Discussion}

Infrared spectroscopy gives a qualitative idea on the adsorption process. Figure 2 shows the infrared spectra using the ATR technique between 650 and $4000 \mathrm{~cm}^{-1}$ of the hydrated Na-montmorillonite, $\mathrm{C}_{10} \mathrm{E}_{3}$-organoclay and $\mathrm{C}_{10} \mathrm{E}_{3}$ in a lamellar phase (bulk $\mathrm{C}_{10} \mathrm{E}_{3}$ ). Both spectra were normalized with respect to the most intense absorption band corresponding to the $\mathrm{SiO}$ stretching mode at $1030 \mathrm{~cm}^{-1}$. The Na-montmorillonite spectrum revealed several bands at 840 , 890 , and $918 \mathrm{~cm}^{-1}$ assigned to the $\mathrm{AlMgOH}, \mathrm{AlFeOH}$ and $\mathrm{AlAlOH}$ deformations, respectively. The broad and intense band at $1030 \mathrm{~cm}^{-1}$ and the shoulder at $1117 \mathrm{~cm}^{-1}$ correspond to the $\mathrm{SiO}$ stretching mode. The $\mathrm{OH}$ angular deformation is observed at $1635 \mathrm{~cm}^{-1}$, whereas the $\mathrm{OH}$ stretching of water spreads out on a wide range of wavenumbers between 3200 and $3600 \mathrm{~cm}^{-1}$. The last broad band at $3626 \mathrm{~cm}^{-1}$ is assigned to the $\mathrm{OH}$ stretching of structural hydroxyl groups. Moreover of the assignment of the bands of the Na-montmorillonite, the spectrum reveals bands characterizing another crystalline or amorphous phases. Indeed, the weak band at $800 \mathrm{~cm}^{-1}$ is assigned to the $\mathrm{SiO}$ stretching of quartz and silica.

The spectrum of $\mathrm{C}_{10} \mathrm{E}_{3}$-organoclay reveals expected bands of the Na-montmorillonite with two additional bands related to the adsorption of $\mathrm{C}_{10} \mathrm{E}_{3}$ onto the clay mineral surface. Indeed, the broad infrared absorption band at $1470 \mathrm{~cm}^{-1}$ is relative to the $\mathrm{CH}_{2}$ scissoring mode whereas the intense absorption bands at 2920 and $2850 \mathrm{~cm}^{-1}$ correspond to the antisymmetric and symmetric $\mathrm{CH}_{2}$ stretching mode of the hydrocarbon chains, respectively ${ }^{12,13}$.

The intensities of both symmetric and antisymmetric $\mathrm{CH}_{2}$ stretching bands are recognized to strongly depend on the density of confined surfactant within the interlayer space ${ }^{12,13}$. Figure 3 shows the infrared spectra of the $\mathrm{C}_{10} \mathrm{E}_{3}$-organoclay, Na-montmorillonite and pure nonionic surfactant recorded in the spectral range of $2800-3000 \mathrm{~cm}^{-1}$. The spectrum of the synthesized organoclay shows a strong intensity for both $\mathrm{CH}_{2}$ stretching bands, underlining the well affinity of $\mathrm{C}_{10} \mathrm{E}_{3}$ with the clay surface and its confinement within the interlayer

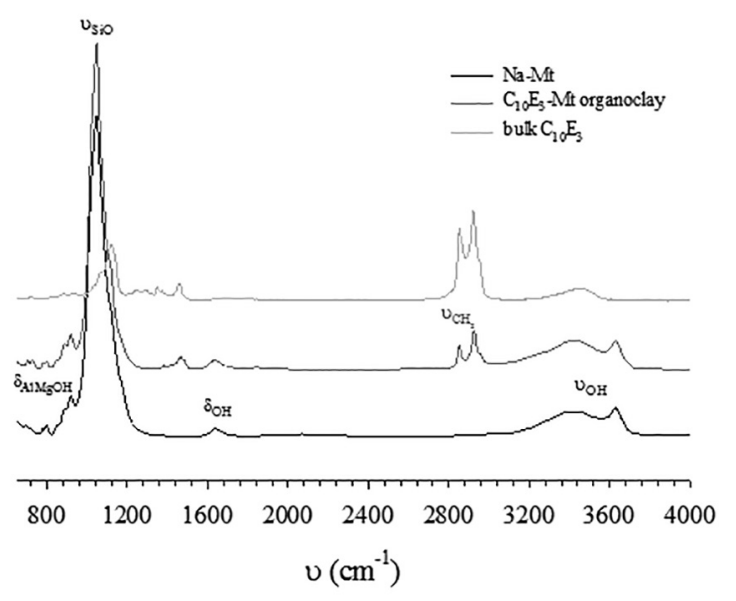

Figure 2: FTIR spectra of Na-montmorillonite (in black solid line), $\mathrm{C}_{10} \mathrm{E}_{3}$-organoclay (dark gray solid line) and pure nonionic surfactant (gray solid line) recorded between 650 and $4000 \mathrm{~cm}^{-1}$. The spectra resolution is $2 \mathrm{~cm}^{-1}$. The $\mathrm{AlXOH}$ ( $\mathrm{X}$ can be $\mathrm{Fe}, \mathrm{Al}$ or $\mathrm{Mg}$ ), $\mathrm{OH}$ deformation and stretching bands are labeled. Moreover, the $\mathrm{CH}_{2}$ stretching of the $\mathrm{C}_{10} \mathrm{E}_{3}$ nonionic surfactant is labeled.

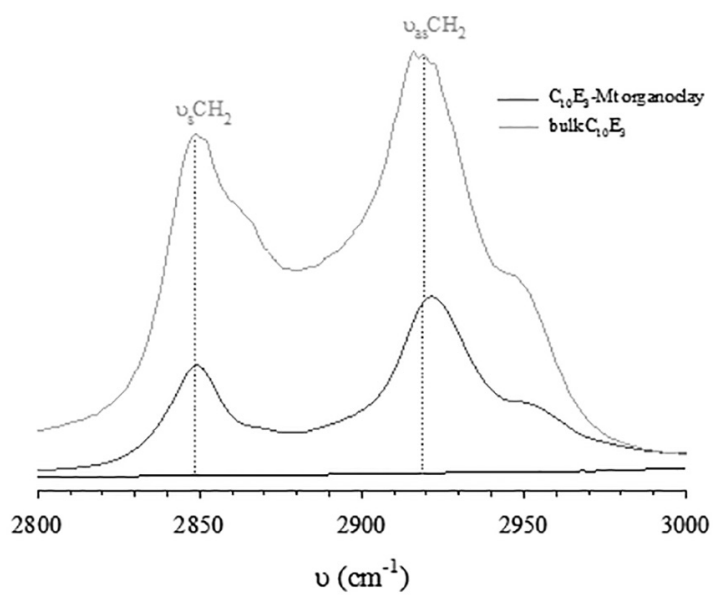

Figure 3: FTIR spectra of (in black solid line), $\mathrm{C}_{10} \mathrm{E}_{3}$-organoclay (dark gray solid line) and pure nonionic surfactant (gray solid line). The symmetric and antisymmetric $\mathrm{CH}_{2}$ stretching of which frequencies indicate the presence of disorder/order in the alkyl chains corresponds to the two bands. The vertical dashed line highlights the small shift in frequencies of the $\mathrm{CH}_{2}$ stretching bands for confined $\mathrm{C}_{10} \mathrm{E}_{3}$.

space. Indeed, moreover to give an idea of the density of confined $\mathrm{C}_{10} \mathrm{E}_{3}$, the wavenumbers of $\mathrm{CH}_{2}$ stretching bands stress out the conformational order of surfactant. For the pure $\mathrm{C}_{10} \mathrm{E}_{3}$, in which the molecules are self-organized in ordered layers, the $\mathrm{CH}_{2}$ stretching bands appear at 2849 and $2918 \mathrm{~cm}^{-1}$, underlining that alkyl chains adopt an all-trans conformation $^{16,17}$. The wavenumbers of both symmetric and antisymmetric absorption bands for the confined surfactant are very close to those of bulk surfactant. This indicates that confined $\mathrm{C}_{10} \mathrm{E}_{3}$ adopt an all-trans conformation within the interlayer space of the clay. 
The X-ray diffraction patterns of the Na-montmorillonite and $\mathrm{C}_{10} \mathrm{E}_{3}$ organoclay are shown in Figure 4. The diffractogram of the Na-montmorillonite displays a broad $(00 l)$ reflections located around $q \sim 0.52,1.06$ and $2 \AA^{-1}$ leading to an interbasal spacing close to $12 \AA$ which underlines the hydration of one water monolayer in the clay according to previous observations and numerical simulations on natural montmorillonites ${ }^{18,19}$. The Na-montmorillonite is also characterized by several diffraction peaks at $q \sim 1.41,2.46$ and $4.19 \AA^{-1}$ corresponding to $(02.11),(20.13)$ and $(06.33)$ reflections, respectively. The Na-montmorillonite sample, despite the purification process, contains quartz impurity, identified with Bragg peaks located at $q \sim 1.48$ and $2.23 \AA^{-1}$.

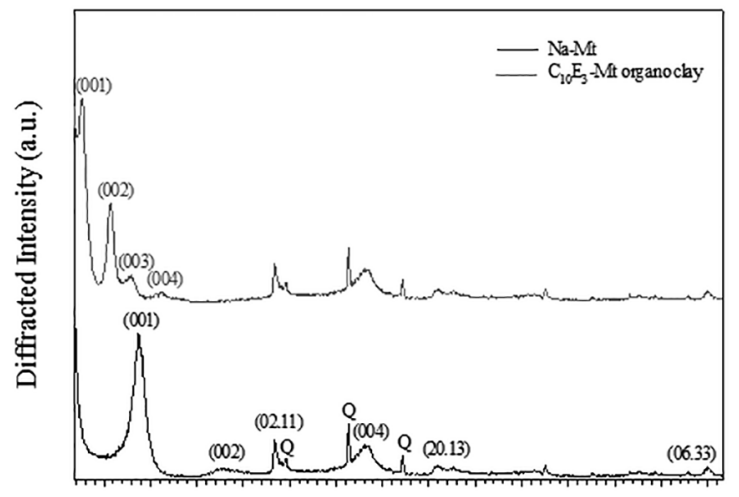

$\begin{array}{llllllllllllll}0.3 & 0.6 & 0.9 & 1.2 & 1.5 & 1.8 & 2.1 & 2.4 & 2.7 & 3.0 & 3.3 & 3.6 & 3.9 & 4.2\end{array}$ $\mathrm{q}\left(\AA^{-1}\right)$

Figure 4: X-ray diffraction patterns of the Na-montmorillonite (in black solid line) and $\mathrm{C}_{10} \mathrm{E}_{3}$-organoclay samples (in dark gray solid line) performed at room temperature. The $(001)$ reflections and (02.11), (20.13) and (06.33) reflections of Na-montmorillonite are labeled. The label Q indicates the presence of quartz in both samples.

The X-ray diffraction patterns of the $\mathrm{C}_{10} \mathrm{E}_{3}$-organoclay show several diffraction peaks at the $q$ located at $0.163,0.34$, 0.48 and $0.66 \AA^{-1}$. It is interesting to remark that $q$ values correspond to $q_{4} \sim 4 q_{1}, q_{3} \sim 3 q_{1}$ and $q_{2} \sim 2 q_{1}$, which reflects the existence of a well ordering in the silicate layers. This demonstrates that the intercalation of nonionic surfactant is stable and $\mathrm{C}_{10} \mathrm{E}_{3}$ forms and ordered structure within the silicate layers. The insertion of $\mathrm{C}_{10} \mathrm{E}_{3}$ expands the distance between the layers, giving a $\mathrm{d}_{001}$ spacing of $\sim 38 \AA$, corresponding to an interlayer separation of $\delta=38-9.7=28.3 \AA$, where 9.7 $\AA$ represents the thickness of the clay layer ${ }^{18,19}$. This value implies that the inserted surfactant must adopt a perpendicular position between the clay surfaces.

More interestingly, the intersheet separation value is very close to that of the $27.6 \AA$ bilayer thickness of the $\mathrm{C}_{10} \mathrm{E}_{3}$ lamellar phase. Another piece of important information derived from XRD pattern of organoclay was the absence of reflection peaks of the $\mathrm{C}_{10} \mathrm{E}_{3}$ in the $q$ region $7.11 \times 10^{-2}-4.3$ $\AA^{-1}$. This indicates that $\mathrm{C}_{10} \mathrm{E}_{3}$ molecules were not aggregated on the external clay surfaces and were mainly intercalated in the interlayer space. Electron density distribution analysis in Figure 5 which represents the electron density distribution of the organoclay, estimated by the relation of $\rho(x)$ against $x$ (Equation (2)), supports the view that organoclay consists of alternatively stacked units of a bilayer sandwiched by clay platelets ${ }^{20}$.

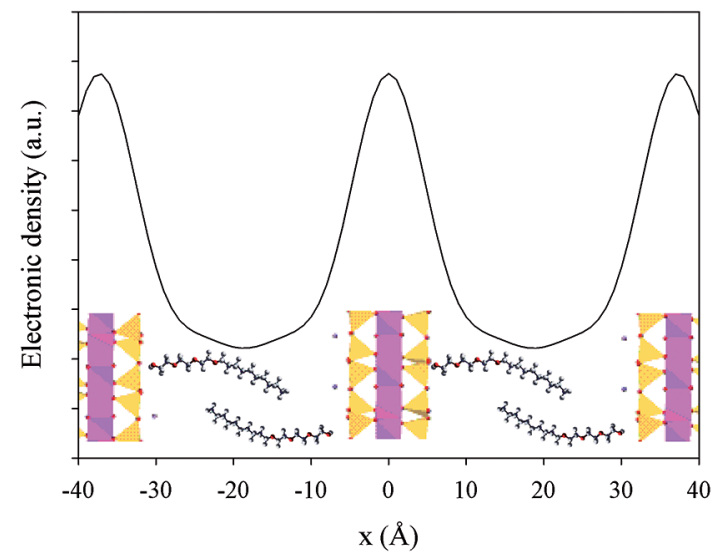

Figure 5: Electron density distribution of $\mathrm{C}_{10} \mathrm{E}_{3}$-organoclay synthesized in which the $\mathrm{C}_{10} \mathrm{E}_{3}$ nonionic surfactant is in lamellar phase of which bilayer thickness is close to $27.6 \AA$. The insert in this figure shows a schematic representation of confined $\mathrm{C}_{10} \mathrm{E}_{3}$ bilayer within the interlayer space of the phyllosilicate layers where the octahedral and tetrahedral layers are in yellow and pink colors, respectively.

Previous studies using nonionic surfactants such as Brij 56 (polyoxyethylene (10) cetyl ether) and polyethylene glycol ether pointed out the adsorption process takes place mainly below but near the CMC and involves a condensation of monomers that leads to a confinement of two molecular layers parallel to the silicate surface ${ }^{11}$. Here, the large expansion of the interlayer space results to the difference of preparation in organoclay where the adsorption of a nonionic surfactant was achieved in a lamellar phase in contrast to previous studies using surfactant at concentration below CMC. Thus, the adsorption of $\mathrm{a}_{10} \mathrm{E}_{3}$ drives to the condensation of a lamellar phase, which generates a hydrophobic environment and expands the interlayer space at large values. Analysis of the supernatant (not shown here) revealed that no sodium were expelled pointing that intercalation of the surfactants preserve the compensating cations within the interlayer space of the clay mineral. Since these cations are kept and nonionic surfactants are intercalated in a normal bilayer or paraffin arrangement, the resulting organoclay displays a dual hydrophilic/hydrophobic behavior which enlarges the possibilities of adsorption for organic compounds ${ }^{8,9}$.

\section{Conclusions}

The intercalation of organic compounds within the interlayer space of natural clay mineral allows the preparation of organoclay nanocomposites for various applications 
(environment, cosmetics, paints, polymers). Organoclay materials are mainly synthesized through ion-exchange with the exchangeable cations in the interlayer space. Another way of synthesis is the use of nonionic surfactants allowing one to preserve the charge characteristics (i.e. the compensating cations) of the starting clay mineral.

In this study, the preparation of the organoclay was achieved when the $\mathrm{C}_{10} \mathrm{E}_{3}$ self-assembles in a lyotropic lamellar phase which leads to the confinement of a bilayer arrangement of $\mathrm{C}_{10} \mathrm{E}_{3}$ within the interlayer space of a Namontmorillonite. Thus, such nonionic organoclay opens the way to new perspectives for the synthesis of hybrid layered nanomaterials since cation exchanges can still be undertaken with the possibility to incorporate functional organic molecules of important size due to the maximum opening of the basal spacing hydrophobicity of the organoclay.

\section{References}

1. Paiva LB, Morales AR, Valenzuela Díaz FR. Organoclays: Properties, preparation and applications. Applied Clay Science. 2008;42(1-2):8-24.

2. Ruiz-Hitzky E, Aranda P, Darder M, Rytwo G. Hybrid materials based on clays for environmental and biomedical applications. Journal of Materials Chemistry. 2010;20(42):9306-9321.

3. Ruiz-Hitzky E, Aranda P, Darder M, Ogawa M. Hybrid and biohybrid silicate based materials: molecular vs. blockassembling bottom-up processes. Chemical Society Reviews. 2011;40(2):801-828.

4. Sposito G, Prost R. Structure of water adsorbed on smectites. Chemical Reviews. 1982;82(6):553-573.

5. Pinnavaia TJ. Intercalated clay catalysts. Science. 1983;220(4595):365371.

6. Thiebault T, Guégan R, Boussafir M. Adsorption mechanisms of emerging micro-pollutants with a clay mineral: Case of tramadol and doxepine pharmaceutical products. Journal of Colloid and Interface Science. 2015;453:1-8.

7. Carli LN, Daitx TS, Guégan R, Giovanela M, Crespo JS, Mauler RS. Biopolymer nanocomposites based on poly(hydroxybutyrateco-hydroxyvalerate) reinforced by a non-ionic organoclay. Polymer International. 2015;64(2):235-241.

8. Guégan R, Giovanela M, Warmont F, Motelica-Heino M. Nonionic organoclay: A 'Swiss Army knife' for the adsorption of organic micro-pollutants? Journal of Colloid and Interface Science. 2015;437:71-79.
9. De Oliveira T, Guégan R, Thiebault T, Le Milbeau C, Muller F, Teixeira V, et al. Adsorption of diclofenac onto organoclays: Effects of surfactant and environmental ( $\mathrm{pH}$ and temperature) conditions. Journal of Hazardous Materials. In press. 2016. doi: 10.1016/j.jhazmat.2016.05.001.

10. Lai MC, Chang KC, Huang WC, Hsu SC, Yeh JM. Effect of swelling agent on the physical properties of PET-clay nanocomposite materials prepared from melt intercalation approach. Journal of Physics and Chemistry of Solids. 2008;69(5-6):1371-1374.

11. Deng Y, Dixon JB, White GN. Intercalation and surface modification of smectite by two non-ionic surfactants. Clay and Clay Minerals. 2003;51(2):150-161.

12. Guégan R. Self-assembly of a non-ionic surfactant onto a clay mineral for the preparation of hybrid layered materials. Soft Matter. 2013;9(45):10913-10920.

13. Guégan R. Intercalation of a nonionic surfactant $\left(\mathrm{C}_{10} \mathrm{E}_{3}\right)$ bilayer into a Na-montmorillonite clay. Langmuir. 2010; 26(24):19175-19180.

14. Guégan R, Sueyoshi K, Anraku S, Yamamoto S, Miyamoto N. Sandwich organization of non-ionic surfactant liquid crystalline phases as induced by large inorganic $\mathrm{K}_{4} \mathrm{Nb}_{6} \mathrm{O}_{17}$ nanosheets. Chemical Communications. 2016;52(8):1594-1597.

15. Guégan R. Confinement effects on water structure in membrane lyotropic phases. Journal of Colloid and Interface Science. 2011;358(2):485-490.

16. Li Y, Ishida H. A differential scanning calorimetry study of the assembly of hexadecylamine molecules in the nanoscale confined space of silicate galleries. Chemistry of Materials. 2002;14(3):1398-1404.

17. Li Y, Ishida H. Concentration-dependent conformation of alkyl tail in the nanoconfined space: hexadecylamine in the silicate galleries. Langmuir. 2003;19(6):2479-2484.

18. Karaborni S, Smit B, Heidug W, Urai J, van Oort E. The swelling of clays: molecular simulations of the hydration of montmorillonite. Science. 1996;271(5252):1102-1104.

19. Ferrage E, Lanson B, Sakharov BA, Drits VA. Investigation of smectite hydration properties by modeling experimental X-ray diffraction patterns: Part I. Montmorillonite hydration properties. American Mineralogist. 2005;90(8-9):1358-1374.

20. Itoh T, Shichi T, Yui T, Takahashi H, Inui Y, Takagi $\mathrm{K}$. Reversible color changes in lamella hybrids of poly(diacetylenecarboxylates) incorporated in layered double hydroxide nanosheets. The Journal of Physical Chemistry B. 2005;109(8):3199-3206. 